\title{
Experiences of Educational Use of Technologies a Case of Facebook, Google Plus and the Use of Blogs
}

\author{
Ana Kennedy Russell \\ Escuela de Ciencias del Lenguaje: Área de Inglés \\ Instituto Tecnológico de Costa Rica \\ Cartago, Costa Rica \\ Email: akennedy@itcr.ac.cr
}

\begin{abstract}
This workshop considers to share some experiences of educational practice of technologies used in English courses at the Technological Institute of Costa Rica, known in Spanish by its abbreviation (ITCR) throughout three years. There are three cases chosen using technological tools such as Facebook, Google Plus and the blog. Although courses at the ITCR are face to face, some technological components have been included in our courses so as to assure a transformation process of both professors and students' roles.

These experiences will be shared during the Eleventh Latin American Conference on Learning Objects and Technologies (LACLO 2016). Once experiences have been public, the aim will be that of motivating professors to make further research pertaining other technological tools which hopefully will permit our students to improve their abilities and performance in the English language.
\end{abstract}

\section{INTRODUCCIÓN}

El Instituto Tecnológico de Costa Rica, conocido por sus siglas como (ITCR), es una universidad de corte científico y tecnológico. No obstante, al igual que todo centro de educación superior serio y de renombre, considera dentro de su malla curricular las ciencias exactas y demás ciencias conexas, así las ciencias sociales y las comunicativas contribuyen a la formación de un profesional más íntegro y acorde con las exigencias de una sociedad más tecnológica, equitativa e inclusiva.

Como parte de las ciencias de la comunicación, el instituto cuenta con la Escuela de Ciencias del Lenguaje, facultada para impartir cursos de servicio en comunicación oral, escrita y técnica, así como cursos de idiomas, principalmente para el aprendizaje y consolidación del inglés. En consecuencia, las experiencias que se comparten se vienen desarrollando en búsqueda de mejorar el desempeño del idioma inglés en el estudiantado que debe matricular dos o más cursos de esta lengua, para su consecuente incorporación al mundo laboral. Dicha inserción estará garantizada por las facultades conferidas por las escuelas involucradas en este proceso de enseñanza-aprendizaje.

A pesar del componente presencial que caracteriza nuestros cursos, se pretende ir incursionando en la metodología de clase invertida; por consiguiente, nuestras lecciones desarrollan un componente virtual que le permite al estudiante ser autodidacta, lo cual resulta en un empoderamiento de su propio proceso educativo. Como resultado, los roles tanto del profesorado como del estudiantado se ven redefinidos. Mientras el docente facilita los espacios de conocimiento y los establece en un repositorio u otros sitios, el alumno selecciona e implementa los conocimientos y los transforma de acuerdo con su nivel de criticidad y con su necesidad.

Los cursos impartidos en inglés pretenden transformar los escenarios de formación, de tal forma que se busca capacitar al profesorado en la mejora de la pedagogía implementada, a partir de la necesidad de potenciar nuestras competencias tecnológicas. Es así como los docentes nos hemos dado a la tarea de buscar una actualización permanente de contenidos, basados en la evolución tecnológica que se va dando. Para tal efecto, pretendo compartir las experiencias del empleo de las herramientas Facebook, Google Plus y el blog, las cuales se utilizaron durante los cursos impartidos en un periodo de 3 años.

Los cursos donde se han empleado estas herramientas van desde un inglés básico hasta los impartidos a través del Consejo Nacional de Rectores (CONARE), de nivel XIII. Con este trabajo, se pretende visualizar las desventajas y sobre todo las ventajas que tanto profesores como estudiantes han experimentado al ser parte de esta novedosa experiencia educativa.

\section{ESTADO DEL ARTE}

Para una mayor comprensión de los términos utilizados durante esta experiencia, se definen algunos términos relacionados con el proceso de educación virtual y lo que concierne a esta metodología de enseñanza- aprendizaje.

Los términos virtual y presencial se definen a partir de experiencias pedagógicas. De manera que es necesario acogernos a la definición que señalan [1], quienes dicen que para efectos docentes la virtualidad corresponde al ambiente provisto de posibilidades comunicativas, informáticas y constructivas que benefician las actividades presenciales de los participantes. Así pues, tanto estas como las actividades virtuales se relacionan a tal grado que estas últimas pasan a ser el sitio y el medio con los cuales se da la primera. 
La tecnología ha provocado un cambio irreversible en el mundo que nos rodea, de ahí que sea necesario incorporarlas a la educación [2] y [3].

Algunas de las herramientas más usadas dentro de la pedagogía son los correos electrónicos, Slideshare, Flickr, Wiki, Cloud, Multimedia, Tumbr, Pinterest, Twiter, Facebook, Google Plus y los blogs, entre muchos otras.

Para [4], las plataformas virtuales son consideradas en algunas instancias como "contenedores de cursos" que adicionan componentes de comunicación y permiten el seguimiento del aprendizaje del estudiantado, mientras que en [5] estas se consideran como espacios donde ocurre el desarrollo del aprendizaje. Finalmente, [4] expresa que para otras son una secuencia de actividades.

Una de las herramientas que escojo compartir es un blog. De esta, [6] da una definición un tanto simplista, pues lo expone como un espacio personal de escritura en internet, donde su autor publica artículos o noticias ("post") que pueden contener texto, imágenes e hipervínculos. Los nuevos contenidos se añaden vía web desde el propio navegador y sin necesidad de otro programa auxiliar.

Algunas deferencias en cuanto a la definición anterior es la que da [7], en la cual se puede observar que los estudiantes también constituyen una parte muy importante en el desarrollo de este como herramienta para las lecciones. Esto en razón de que el blog procura ser un espacio colaborativo, donde los alumnos realizan diálogos, conversaciones, entrevistas, discusiones $u$ otras actividades que les permitan interactuar y construir conocimiento en equipo. Se añade el hecho de que puede compartirse con Google Plus para potenciar otras actividades, como el uso de videoconferencias o "hangouts", chats en combinación con el blog.

Por otro lado, Google Plus se define por [8]como una red social que permite interactuar con personas y compañías, las cuales se pueden clasificar en círculos de acuerdo con la afinidad o tipo de relación que se mantenga con cada uno de los participantes. En estos, se cataloga cada una de las personas con quienes se tiene contacto y ellas juegan un rol determinado (esto definirá en qué círculo se les incluye). Se podrán crear tantos círculos como se quisiera, con los nombres y significados que se consideren apropiados (no son visibles para los demás) [9].

\section{METODOLOGÍA}

Como estrategia metodológica inicial, antes de implementar el uso de estas herramientas, se aplicó una encuesta. Esta buscaba obtener información sobre las facilidades que presentan los estudiantes de nuevo ingreso en torno al acceso y uso de internet mediante distintos dispositivos.

Para medir la efectividad de las actividades implementadas en las distintas plataformas, se observó la reacción de los estudiantes y su cambio conductual a través de su participación.

Así, se evidenció que los alumnos ante actividades virtuales se sienten con mayor libertad de participar, pues los periodos son más prolongados, mientras que las actividades presenciales se daban únicamente durante las horas lectivas, lo cual constituía una presión temporal para desarrollarlas.

También, se observó que, a pesar de la explicación dada por el profesor sobre el uso del blog, los estudiantes durante el primer uso de Google Plus y en otros casos se mostraron un tanto confusos, pues temían preguntarle al docente sobre el uso de la herramienta.

Hubo una alta participación, lo cual denota el interés del estudiantado en utilizar estas plataformas.

Con el fin de compilar los resultados de estas experiencias, se ha implementado el enfoque metodológico de corte cualitativo; no obstante, también se considera el cuantitativo para efectos de realizar una futura encuesta a los usuarios del blog. Se hace un análisis cualitativo de los resultados obtenidos en las herramientas seleccionadas, sobre todo en cuanto al uso y participación de los estudiantes en el blog. De ahí, se compara el enfoque cuantitativo con el cualitativo, a fin de justificar y aclarar los resultados de las experiencias vividas durante la implementación de las herramientas a exponer.

Se trabajó con estos enfoques metodológicos para recopilar las experiencias adquiridas a lo largo de tres años de emplear herramientas como Facebook, Google Plus y el blog, entre otras, para elaborar material y trabajar tanto dentro como fuera del aula; es decir, virtual y presencial. En este caso, se trabajó con:

- $\quad$ Partes interesadas: Ana Kennedy Russell

- $\quad$ Número de participantes: 30 personas que cuenten con algún dispositivo que tenga cámara y micrófono, con acceso a internet.

\section{RECOPILACIÓN DE EXPERIENCIAS EDUCATIVAS A PARTIR DEL USO DE HERRAMIENTAS TECNOLÓGICAS}

El uso de plataformas que nos permitan compartir conocimiento sin necesidad de la presencia física en el salón de clase es una ventaja de la implementación de las herramientas tecnológicas en general. Se han seleccionado algunas actividades realizadas durante el desarrollo de distintos cursos de inglés, en el marco del uso de herramientas tecnológicas de orden pedagógico.

Con el fin de contar con una referencia sobre el estado de accesibilidad de herramientas tecnológicas por parte de los estudiantes que matricularon algunos cursos de inglés de primer ingreso en el 2013, se les aplicó una encuesta, para la recolección de datos prioritarios ante la posibilidad de crear un laboratorio virtual. De los resultados, se pueden rescatar, para los fines de este estudio, los ítems 6 y 7. 
Dicha encuesta se realizó de forma digital, utilizando la herramienta que proporciona Google para estas. De los 86 estudiantes de nuevo ingreso que la hicieron, se obtuvieron los siguientes resultados para los ítems mencionados. Para el 6 (“¿Cuenta usted con la facilidad de utilizar internet?"), el $97 \%$ respondió afirmativamente, contra un $3 \%$ que lo hace de forma negativa (ver Fig. 1.). Estos datos se emplean para asegurarnos que se nos facilitará la implementación de herramientas tecnologías, ya que se puede dar por un hecho que el acceso a estas plataformas no sería un problema para los estudiantes.

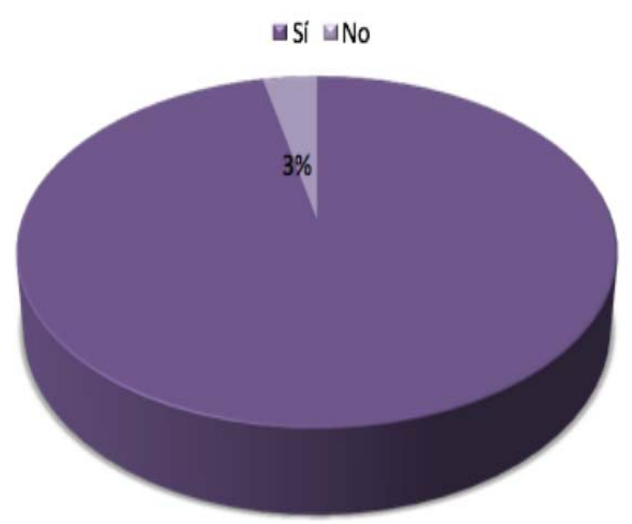

Fig. 1. Gráfico que muestra la respuesta a “ ¿Cuenta usted con la facilidad de utilizar internet?"

Seguidamente, para el ítem 7 ("Si su respuesta es afirmativa, marque la o las opciones que corresponden a su realidad"), se obtuvieron los siguientes resultados: a. $24 \%$ de los encuestados cuentan con un teléfono con internet; $b$. el $26 \%$ tiene un teléfono inteligente; c. solamente un $4 \%$ cuenta con una "tablet"; e. el $13 \%$ dispone de una computadora, la cual es ofrecida por la institución donde llevan los cursos; f. tan solo el $2 \%$ cuenta con una computadora donde labora y g. el $7 \%$ tienen una computadora en la familia (ver Fig. 2.).

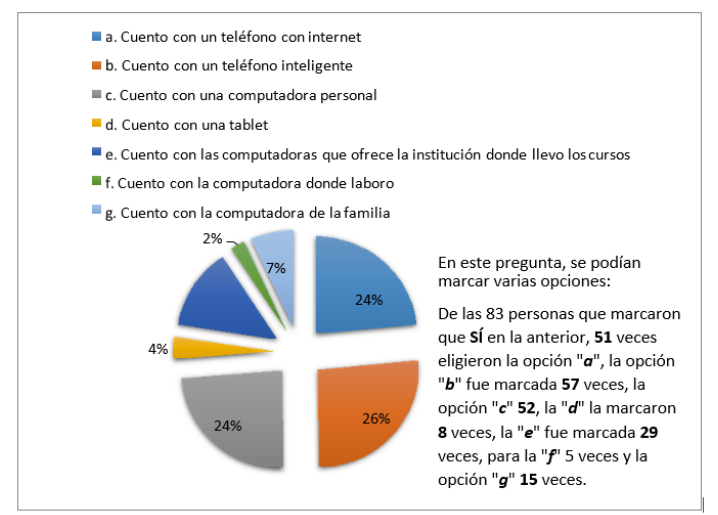

Fig. 2. Gráfico que muestra la respuesta a "Si su respuesta es afirmativa, marque la o las opciones que corresponden a su realidad".
Los resultados de la encuesta indican que la mayoría de los estudiantes de nuevo ingreso cuenta con algún dispositivo que le permite acceder a alguna herramienta tecnológica, al mismo tiempo se obtuvo un panorama un poco más claro acerca del tipo de alumno que matricula los cursos de inglés. A pesar de ello, resultó asombroso encontrarse con estudiantes, sobre todo de primer ingreso, que carecen del conocimiento de muchas herramientas tecnológicas, como lo son Google Plus y el mismo el blog, que permite realizar publicaciones.

A lo largo de esta investigación, se ha logrado determinar que el escaso conocimiento del blog entre algunos estudiantes hace difícil su empleo, a no ser que se les indique utilizarlo para la implementación de alguna actividad.

A parte del Facebook, que es la herramienta más conocida entre el estudiantado, se escogen estas dos, en particular, por la versatilidad y flexibilidad que permiten tanto al docente como al estudiante a la hora de elaborar actividades y ponerlas en práctica. El enlace de la actividad de Facebook y el ya reconocido TEC-Digital se da porque esta última es la principal plataforma implementada para el desarrollo de los cursos en el ITCR.

Al compartir estas experiencias, se pretende que contribuyan a mejorar el proceso de enseñanza aprendizaje, tal y como ha acontecido en los cursos donde tales prácticas se han implementado. Los cursos impartidos donde se han usado estas herramientas tecnológicas son Inglés Básico, Inglés I, Inglés II, Inglés I para Computación, Inglés II para Computación, Inglés III para Computación, Inglés I para la Administración de la Tecnología de la Información, Inglés I para Biotecnología, Inglés II para Biotecnología, Inglés para CONARE del I al XIII y cursos para profesorados que optan por la enseñanza del inglés como lengua extranjera entre otros. Los ejemplos se visualizan en la Fig. 3., como referencias de los nombres de los distintos cursos impartidos a lo largo del periodo establecido.

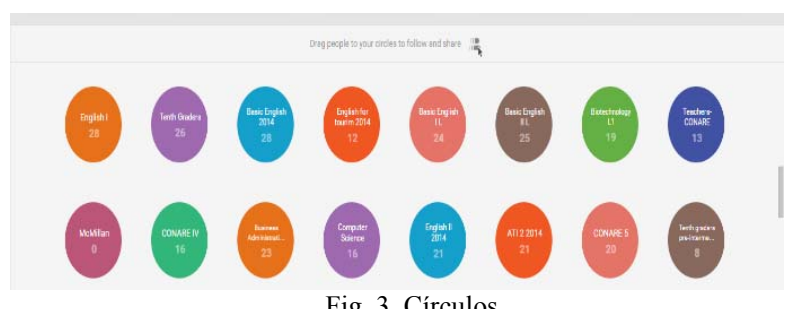

La dinámica implementada durante estos tres años para la puesta en práctica de estas herramientas se ejemplifica por medio de tres casos definidos como: Caso 1: entrevista en Facebook, publicada en el TEC-Digital; Caso 2: Google Plus en combinación con YouTube y el Caso 3: el blog. Para efectos de este taller, cada uno de estos cuenta con una actividad específica, salvo el Caso 3, que tiene tres distintas. Este último refuerza lo planteado en el Estado del Arte, pues 
prueba que la definición del blog es más que un repositorio personal, donde también los estudiantes pueden dialogar y realizar debates; en fin, participar en actividades colaborativas, las cuales buscan contribuir a la construcción de un conocimiento significativo, según sus propias necesidades. Al igual que el caso 3, los otros también contienen una descripción de la actividad junto con las instrucciones a seguir, el objetivo que se alcanzó, los resultados obtenidos y las percepciones que el profesor obtiene a partir del comportamiento, así como del proceso de la realización de las distintas actividades.

\section{A. Caso \#1: El uso del Facebook, publicado en el TEC- Digital}

Descripción de la actividad:

Para esta actividad, se solicitó a los estudiantes escoger una herramienta que les facilitara video-grabarse en una entrevista o poder realizar captura de pantallas de un chat que se realizara; este último debería ser una entrevista con algún conocido que tuviera el mismo nivel de dominio del inglés que el estudiante, o bien, superior. Luego de videograbarse o de realizar las respectivas capturas de pantalla de la entrevista, debeían subirla al TEC- Digital. Todos escogieron utilizar el Facebook para localizar personas fuera del aula que pudieran conversar en inglés; en caso de no tener conocidos, podían escoger a algún compañero del aula o de otro curso. Para esta experiencia, se asignó a los estudiantes un tema de conversación. La actividad parte de que ellos debían elaborar preguntas de información general, con el fin de entrevistar a esa persona en inglés; como mínimo, debieron confeccionar 10 preguntas para que las respondiera el entrevistado y luego se cambiaba el rol. Los participantes podían escoger entre dos formas para entregar su entrevista: escribir en un chat en la plataforma o grabar un video con la entrevista que se pudiera escuchar y subirlo al TEC- Digital (ver Fig. 4.).

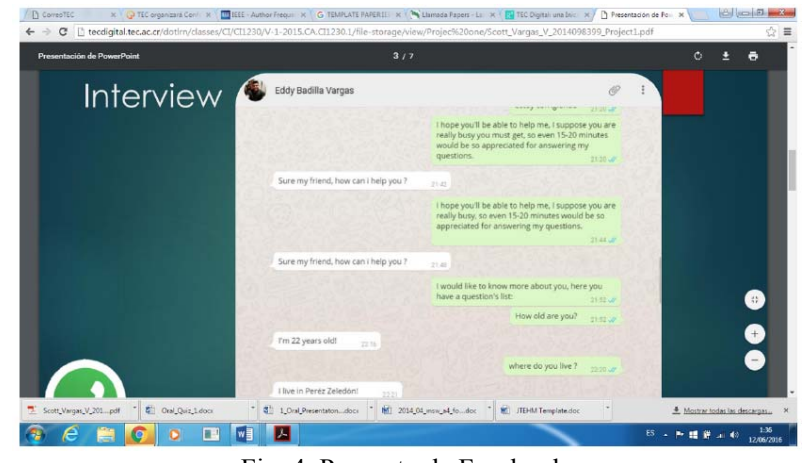

Fig. 4. Proyecto de Facebook.

Objetivo:

Utilizar el presente simple y preguntas de información en este tiempo verbal.

\section{Resultado:}

El resultado fue de mucho provecho, ya que los estudiantes escogieron Facebook y subieron al TEC-Digital una captura de pantalla del chat y enviaron a mi correo los videos elaborados. Los trabajos fueron realizados desde los teléfonos celulares, laptops o computadoras, en algunos casos en las del laboratorio de la institución disponibles para el estudiantado.

\section{Percepciones:}

Los alumnos se sintieron bastante cómodos con esta actividad por dos razones: escogieron la plataforma con la que estaban familiarizados y porque eligieron o elaboraban las preguntas para la entrevista.

\section{B. Caso \#2: El uso de Google Plus en combinación con YouTube}

Para esta actividad, el docente convoca a los estudiantes a conectarse a cierta hora en un "hangout", para poder ver un video por medio de YouTube. A finalizar este, la profesora implementa el "hangout", con el fin de aplicarles de forma oral algunas preguntas de comprensión del video.

\section{El objetivo:}

Implementar por medio de un "hangout", en Google Plus, una actividad de comprensión auditiva.

\section{Resultado:}

Se emplea Google Plus en combinación con YouTube como repositorio de materiales y como plataforma para realizar interacciones entre estudiantes, como las conversaciones, discusiones y debates sobre diversos temas. También, se implementa la práctica colaborativa y el empoderamiento del estudiantado al tener que leer artículos, escoger ejercicios y hacer quices para mejorar su desempeño en el idioma.

\section{Percepciones:}

El uso de Google también se ha implementado en los cursos para realizar chat en inglés y ejercicios de comprensión auditiva con videos bajados de YouTube, donde los estudiantes logran ver estos de forma simultánea. La Fig. 5. permite visualizar los "hangout". 


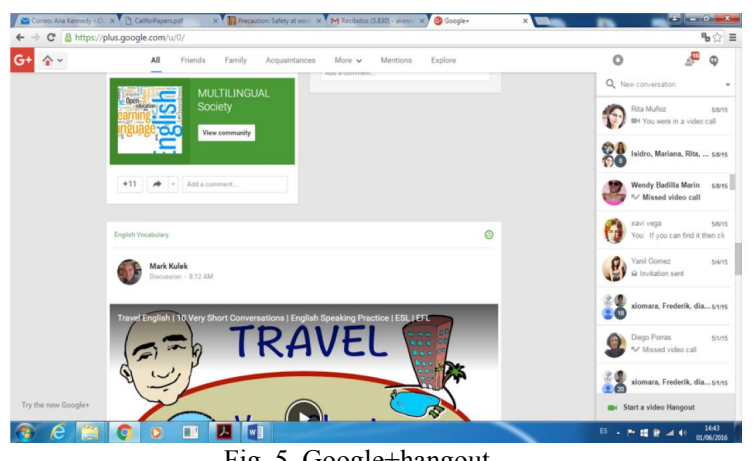

Fig. 5. Google+hangout.

Además, se contó con más de 60 grupos o círculos trabajados a lo largo de 3 años, desde el segundo semestre del 2013 a la fecha. En la Fig. 3., se pueden ver los círculos.

\section{Caso \#3: El uso del blog}

La creación de este blog por parte de la profesora Ana Kennedy nace con la idea de que este no solo sea un repositorio de materiales didácticos, sino que los estudiantes puedan participar activamente; siempre, se debía mantener el respeto para todos los participantes y realizar intervenciones en inglés sobre temas de toda índole. Este blog está entrelazado con Google Plus, de modo que se utiliza como un repositorio, así como una plataforma para realizar ejercicios interactivos, postear tareas, proyectos y pruebas cortas, y hacer tanto prácticas como todo tipo de pruebas escritas. Este blog está creado para los alumnos que van cursando los distintos niveles; es decir, las actividades diseñadas o colocadas en esta herramienta se irán desarrollando según las necesidades de aprendizaje de cada grupo de estudiantes, siempre manteniendo el perfil de ingresos de estos.

Para efectos de compartir experiencias, se han escogido dos casos: el Caso 3.1 (How to save our transportation system?) y el 3. 2 (The Devil Dresses Prada).

Se ha compartido un "role play" entre estudiantes de diversos grupos que se encuentra al mismo nivel de aprendizaje como entre aquellos de distintos cursos para constatar si la participación de un grupo sería igual, mayor o menor al otro. Para ello, se define en casos y se le asigna el nombre de la actividad que aparece en el blog.

\section{Caso 3.1. del blog}

Este lo llamaremos por su nombre en el blog: www.precautionatwork.blogspot.com.

$$
\begin{aligned}
& \text { What problems do we have in Costa Rica } \\
& \text { regarding the transportation system? } \\
& \text { How would you improve the transportation } \\
& \text { system of our county? }
\end{aligned}
$$

Fig. 6. Interrogantes iniciales del www.precautionatwork.blogspot.com.

\section{Descripción del caso:}

En este caso, se le pidió a una compañera que impartió Inglés III para Administración de Empresas (B1-) que compartiera una actividad de "role play" con un curso que se impartió de Inglés III para Computación (B+). Conscientes de que ambas nomenclaturas corresponden a bandas asignadas por nivel, según el Marco Común Europeo, y que son levemente distintas, procedimos a implementar la actividad en el blog.

\section{Descripción de la actividad:}

La actividad consistió en asignarle un "role play" diferente a cada grupo según su perfil de entrada.

Uno de los temas que se debía tratar en ambos cursos era el de transporte público. En virtud de que los estudiantes ya manejaban conceptos básicos de los distintos medios de transporte, se buscó realizar una actividad más retadora, donde el alumno debiera ser crítico a la hora de plantear soluciones a los problemas dados. Entonces, se definió que el estudiante debía escoger un problema, formulado como pregunta, para buscarle la mejor solución. Esta actividad es un ejemplo de cómo el conocimiento adquirido se utiliza en una situación concreta para obtener un resultado; es decir, la solución a un problema.

Los temas fueron: ¿Cómo salvar nuestro sistema de transporte?

\section{How to save our transportation system?}

Fig. 7. Tema, www.precautionatworkblogspot.com.

\section{Instrucciones}

Las instrucciones fueron dadas en inglés de la siguiente forma:

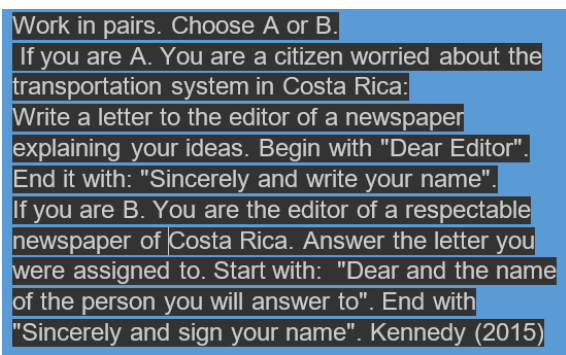

Fig. 8. Instrucciones, www.precautionatwork.blogspot.com.

Adicional a las instrucciones, se les colocó en el blog una carta, en la cual se indicaba las partes que esta debiera contener, finalmente se agregó un link para que pudieran leer sobre distintos tipos de cartas y ver un video al respecto: 
If you wish to learn more about how to write

different kinds of letters, watch the video or

read the information on this link:

http://www.goodletterwriting.com/formalletters.html

Fig. 9. Datos adicionales, www.precautionatwork.blogspot.com.

Para visualizar la participación de los estudiantes en esta actividad, se puede ingresar a la siguiente dirección del blog: http://precautionatwork.blogspot.com/search?q=transportatio $\underline{\mathrm{n}}$

\section{El objetivo:}

Percibir problemas de transporte en su comunidad y plantear soluciones a estos.

\section{Resultado:}

Con esta actividad, se logró un trabajo colaborativo entre los distintos estudiantes, sin menospreciar que fueran de distintos cursos de inglés.

\section{Percepciones:}

Los alumnos que participaron lograron el objetivo señalado. De igual manera, esta actividad les permitió adquirir destrezas en cuanto a la elaboración de distintos tipos de cartas, así como en el vocabulario pertinente para el planteamiento de problemas y soluciones.

\section{Caso 3.2. del blog}

Este lo llamaremos por su nombre en el blog: www.precautionatwork.blogspot.com

\section{The Devil Dresses Prada}

Fig. 9. Nombre del blog, www.precautionatwork.blogspot.com.

\section{Descripción del caso:}

En este caso, se le pidió a una compañera que impartió Inglés II para Administración de empresas (A2+) que compartiera una actividad de discusión con el grupo de Inglés III para Administración de Empresas (B1-) asignadas por nivel, según el Marco Común Europeo, y que son levemente distintas. Por lo tanto, procedimos a implementar la actividad en el blog.

\section{Descripción de la actividad:}

Se les pidió a los estudiantes ver un video sobre una empresa de ropa. Se les asignaron dos preguntas a dos alumnos, uno de cada grupo, para que debatieran sobre la respuesta que consideraba adecuada; en este caso, no existe ninguna respuesta incorrecta.

Uno de los temas que se debía tratar en ambos cursos era el de una empresa exitosa.
En virtud de que los estudiantes ya manejaban conceptos básicos de sobre prendas de vestir, se buscó resaltar la criticidad a la hora de que los participantes dieran sus respuestas.

\section{Instrucciones:}

Estas son las instrucciones dadas para la actividad del video:

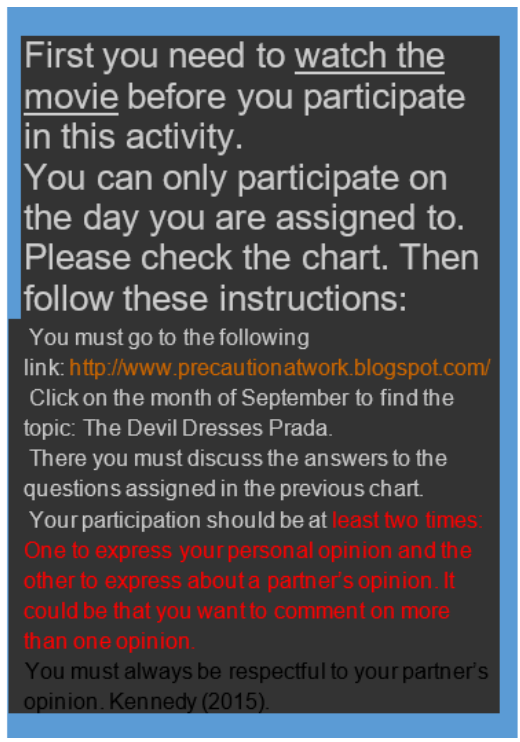

Fig. 10. Actividad del blog.

Las preguntas fueron colocadas en un archivo en el TECDigital y en el blog.

Para ver la participación de los estudiantes en esta actividad, se puede ingresar a la siguiente dirección: http://precautionatwork.blogspot.com/2015/09/the-devildresses-prada.html

El objetivo:

Discutir y sobre las empresas y personaje exitosos.

Resultado:

Con esta actividad, se logró un trabajo colaborativo entre los distintos estudiantes, sin importar que fueran de distintos cursos de inglés.

\section{Percepciones:}

Los estudiantes tuvieron cierta dificultad para descargar este video. Sin embargo, una vez que lograron verlo, discutieron en parejas y grupos las respuestas a las interrogantes planteadas.

Las preguntas fueron colocadas en un archivo en el TECDigital y en el blog.

Para ver la participación de los estudiantes en esta actividad, se puede ingresar a la siguiente dirección: http://precautionatwork.blogspot.com/2015/09/the-devildresses-prada.html 


\section{CONCLUSIONES}

De este trabajo, entre las conclusiones se considera, por un lado, las experiencias vividas por parte de los estudiantes, agrupadas en ventajas y desventajas de utilizar las herramientas tecnológicas propuestas, por otro, lo experimentado por el docente, quien participa como observador y guía para emplear las herramientas en cada una de las actividades llevadas a cabo.

\section{A. Ventajas para el estudiante:}

- Permite un enlace con el profesor para evacuar dudas.

- Brinda la posibilidad de un trabajo colaborativo entre estudiantes del mismo grupo.

- Ofrece la oportunidad de realizar un trabajo colaborativo entre alumnos de distintos grupos.

- Admite el trabajo colaborativo de estudiantes con amigos o parientes con cierto nivel de inglés.

- Hace que el estudiante pueda medir su progreso personal.

- Permite al alumno usar herramientas que solo había escuchado mencionar.

- Mantiene los índices de deserción en niveles bastante bajos. Solo dos o tres estudiantes se retiran por diversas razones.

\section{B. Desventajas para el estudiante:}

- Algunas herramientas no son de fácil acceso en ciertas regiones del país.

- A ciertos estudiantes les cuesta adaptarse a algunas plataformas.

- Surgen inconvenientes de índole técnico (fallas en internet o en el fluido eléctrico, entre otros).

C. Ventajas para el docente:

- Permite integrar elementos educativos para fomentar en el estudiantado el autoaprendizaje.

- Fomenta en el estudiantado la colaboración.

- Brinda la posibilidad de reforzar la reflexión y criticidad entre los estudiantes.

- Puede dar al estudiantado un aprendizaje responsable del empoderamiento de su proceso educativo.

- Da un valor agregado a los contenidos, los cuales por sí solos no motivan al análisis y reflexión.

- Permite en una constante búsqueda del cambio y la consecuente mejora de las estrategias implementadas en el proceso de enseñanza.

\section{Desventajas para el docente:}

- Se debe disponer de tiempo para indagar sobre las posibles herramientas y sus usos.

- Se requiere tiempo para la preparación y estructuración de las lecciones.

\section{RECOMENDACIONES}

El análisis de estas experiencias nos permitirá ejecutar acciones que ayuden a impactar la educación en la era tecnológica, sin dejar al lado la eficacia de las herramientas en uso. En consecuencia, le permitirá al docente hacer una selección de herramientas útiles según a la naturaleza del objeto de aprendizaje. Esto se hará buscando mejorar el desempeño de los estudiantes, al influir positivamente en el proceso de adquisición de conocimiento y habilidades del idioma en cuestión.

Al compartir conocimientos adquiridos sobre el uso de las herramientas seleccionadas y utilizadas durante tres años para complementar el aprendizaje del idioma inglés en estudiantes del Instituto Tecnológico de Costa Rica, se han propuesto algunas ideas de actividades realizables y exitosas con las tres herramientas en cuestión.

Como resultado de la implementación de estas actividades en la virtualidad, se podrá orientar o redireccionar las diversas actividades que se incorporamos en los cursos de la institución para la enseñanza de un segundo idioma.

Razonablemente, en nuestra búsqueda de la excelencia académica, nos tratamos de conectar con herramientas que contribuyan a hacer de nuestro sistema más eficiente al optimizar los recursos disponibles. En este sentido, cuanto más estudiantes y docentes se sirven de estas herramientas, mayor podría ser la posibilidad de ampliar su aprendizaje de forma colaborativa, por ende, su criticidad y análisis.

Al compartir estas experiencias, se intenta motivar a otros profesores a indagar, dentro del marco de las herramientas pedagógicas disponibles, alternativas que resulten eficaces y le permita al estudiantado mejorar tanto su desempeño como habilidades en el idioma que se está adquiriendo.

\section{AGRADECIMIENTOS}

La autora expresa su agradecimiento a los estudiantes que hicieron posible la implementación de las actividades desarrolladas en los distintos cursos. Finalmente, agradece a las compañeras Ingrid Herrera, Patricia Calvo y Mabel Solano por su apoyo, pues como profesoras colaboraron al facilitar la participación de sus alumnos en alguna actividad.

\section{REFERENCIAS BIBLIOGRÁFICAS}

[1] F. R. Dominguez, S. F. Salazar, "et al". La docencia universitaria en los espacios virtuales, San José, Costa Rica: Universidad de Costa Rica, 2009.

[2] C. Pittman, "5 herramientas tecnológicas para mejoras tus clases," 2012. [En línea]. Available:

http://noticias.universia.es/enportada/noticia/2012/08/24/960984/5-herramientastecnológicas-mejorar- clases.html.. 
[3] [En línea]. Available: http://noticias.universia.es/enportada/noticia/2012/08/24/960984/5-herramientastecnologicas-mejorar-clases.html.

[4] J. Sánchez, "Plataformas tecnológicas para el entorno educativo," Acción pedagógica, no 14, pp. 18-24, 2005.

[5] J. Sanchez, 2005. [En línea]. Available: http://www.saber.ula.ve/bitstream/123456789/17239/2/ articulo2.

[6] A. Ramírez, 309 2014. [En línea]. Available: http://www.informaticaparanovatos.com/que-es-unblog/.

[7] A. K. Russel, www.precautionatworkblogspot.com, 2014. [En línea].

[8] "bienpensado Google+ (2016)".

[9] D. Gómez, "http://bienpensado.com/google-plus-quees-y-para-que-sirve/," [En línea]. Available: http://bienpensado.com/google-plus-que-es-y-para-quesirve/. [Último acceso: 2508 2016].

[10] J. I. Ruiz Olabuénaga, Metodología de la Investigación cualitativa, Bilbao, España: Universidad de Deusto, 1999.

[11] "Designing and Developing Software for Educative Virtual Laboratories with Language Processing," 2013.

[12] M. Aravena, E. Kimelman, B. Micheli, R. Torrealba, and J. Zúñiga, Investigación Educativa I, Chile, 2006. 